\title{
Efeito da estratificação em substrato esterilizado na qualidade sanitária de sementes de Ilex paraguariensis
}

\author{
Effect of stratification in sterilized substrate in the sanitary quality of Ilex \\ paraguariensis seeds
}

\section{Gabriela Fernanda Souza ${ }^{I}$, Luciana Magda de Oliveira ${ }^{\mathrm{II}}$, Lenita Agostinetto ${ }^{\mathrm{III}}$, Louise Zago Puchale ${ }^{\mathrm{IV}}$, Alexandra Cristina Schatz Sá ${ }^{\mathrm{V}}$}

\section{Resumo}

\begin{abstract}
A estratificação das sementes de Ilex paraguariensis A. St.-Hill. (erva-mate) em areia úmida por períodos de quatro a seis meses é uma prática comum em viveiros, utilizada para a superação de dormência das mesmas. Entretanto, este processo pode apresentar difícil controle fitossanitário, devido ao elevado grau de umidade e a grande concentração de sementes no mesmo local. Assim, objetivou-se verificar a influência da estratificação em areia autoclavada na qualidade sanitária de sementes de erva-mate. Sementes de quatro lotes foram submetidas à estratificação em areia úmida autoclavada, durante três e seis meses, tendo sua sanidade avaliada em meio BDA ao final destes períodos, assim como em sementes que não passaram por estratificação. Ao final do período de estratificação, as sementes apresentaram aspecto superficial escurecido, o que pode estar ligado à ação dos fungos na degradação do endocarpo. Foram identificados: Fusarium graminearum, Fusarium oxysporum, Fusarium guttiforme, Paecilomyces sp., Aspergillus sp., Penicillium sp. Já Aureobasidium melanogenum, Trichoderma sp., Curvularia sp., Rhizopus sp., Alternaria sp., Cladosporium sp. e Bipolaris sp. tiveram menores ocorrências. Observou-se a redução de Fusarium graminearum, Fusarium oxysporum, Fusarium guttiforme, Aspergillus sp. e Penicillium sp. após a estratificação em areia autoclavada e o gênero Paecilomyces somente foi observado aos 180 dias de estratificação, podendo estar relacionado com a degradação do endocarpo de sementes de Ilex paraguariensis.
\end{abstract}

Palavras-chave: Erva-mate; Superação de dormência; Patologia de sementes

\begin{abstract}
The stratification of Ilex paraguariensis seeds (yerba mate) in wet sand for periods of four to six months is a common practice in nurseries, used to overcome their dormancy. However, this process may present difficult phytosanitary control due to the high moisture content and the high concentration of seeds in the same place. Thus, the present work had the objective of verifying the influence of the stratification in autoclaved sand on the sanitary quality of yerba mate seeds from different lots. Seeds of four lots were submitted to wet stratification for three and six months, and their sanity was evaluated at the end of these periods, as well as seeds that did not undergo stratification. At the end of the stratification period, the seeds had a darkened surface appearance, which may be related to the action of the fungi in the degradation of the endocarp. These ones were identified: Fusarium graminearum, Fusarium oxysporum, Fusarium guttiforme, Paecilomyces sp., Aspergillus sp., Penicillium sp. Already Aureobasidium melanogenum, Trichoderma sp., Curvularia sp., Rhizopus sp., Alternaria sp., Cladosporium sp. and Bipolaris sp. had fewer occurrences. Observed reduction of Fusarium graminearum, Fusarium oxysporum, Fusarium guttiforme, Aspergillus sp. and Penicillium sp. after stratification and the genera Paecilomyces was only observed at 180 days of stratification, and may be related to the degradation of the endocarp of Ilex paraguariensis seeds.
\end{abstract}

Keywords: Yerba mate; Overcoming dormancy; Seed pathology

Engenheira Florestal, MSc., Departamento de Engenharia Florestal, Universidade do Estado de Santa Catarina, Av. Luiz de Camões, 2090, CEP 88520-000, Lages (SC) Brasil. gabisouza93@hotmail.com (ORCID: 0000-0003-3505-5258)

Engenheira Florestal, Dra., Professora do Departamento de Engenharia Florestal, Universidade do Estado de Santa Catarina, Av. Luiz de Camões, 2090, CEP 88520-000, Lages (SC) Brasil. luciana.oliveira@udesc.br (ORCID: 0000-0001-7362-1041)

III Agrônoma. Dra., Professora do Programa de Pós Graduação em Ambiente e Saúde, Universidade do Planalto Catarinense, Av. Castelo Branco, 170, CEP 88509-900, Lages (SC) Brasil. leagostinetto@yahoo.com.br (ORCID: 0000-0002-0468-883X)

Engenheira Florestal, Departamento de Engenharia Florestal, Universidade do Estado de Santa Catarina, Av. Luiz de Camões, 2090, CEP 88520000, Lages (SC) Brasil. louise_zp@hotmail.com (ORCID: 0000-0003-3640-9097)

Engenheira Florestal, Mestranda do Departamento de Engenharia Florestal, Universidade do Estado de Santa Catarina, Av. Luiz de Camões, 2090, CEP 88520-000, Lages (SC) Brasil. alexandra.schatz.sa@gmail.com (ORCID: 0000-0003-1532-8765) 


\section{Introdução}

A dormência de sementes é caracterizada pela ausência de germinação, mesmo em condições ambientais favoráveis, devido a fatores da própria semente, consistindo em um mecanismo de defesa que busca garantir a distribuição da germinação no tempo e no espaço (FOWLER; BIANCHETTI, 2000; MARCOS FILHO, 2005; BEWLEY et al., 2013).

As sementes de erva-mate (Ilex paraguariensis A. St.-Hill.) são classificadas como dormentes e uma prática amplamente utilizada por viveiristas, visando à superação da dormência, é a estratificação das sementes em areia durante períodos de quatro a seis meses, período no qual os embriões rudimentares da espécie podem completar o seu desenvolvimento, assim como possibilitar a permeabilidade do tegumento (ZANON, 1988; DANIEL, 2009; POLETTO et al., 2015).

Em muitos casos, o processo de estratificação apresenta difícil controle fitossanitário, devido ao elevado grau de umidade e à grande concentração de sementes no mesmo local (CROCE; FLOSS, 1999; POLETTO et al., 2015). Grigoletti Júnior e Auer (1996) observaram que sementes estratificadas de erva-mate apresentaram um alto índice de contaminação por fungos, dentre eles do gênero Fusarium. Fatores como a não desinfestação do substrato ou sua reutilização, e o beneficiamento inadequado das sementes (presença de restos de polpa e outras impurezas) favorecem o aparecimento de patógenos, inviabilizando-as ou provocando doenças nas plântulas.

Entretanto, de acordo com Grigoletti Junior et al. (1999), alguns fungos associados às sementes de erva-mate no processo de estratificação apresentam um efeito de degradação do endocarpo. Este fato favorece a germinação das sementes da espécie, auxiliando na superação de dormência física das mesmas.

Com base no exposto, objetivou-se verificar a influência da estratificação em areia autoclavada na qualidade sanitária de sementes de erva-mate de diferentes locais de coleta.

\section{Material e métodos}

Para a realização dos processos de estratificação foram utilizadas sementes de erva-mate provenientes de quatro localidades, sendo que cada local de colheita corresponde a um lote de sementes avaliado (Tabela 1 ).

Tabela 1 - Locais de coleta de sementes de Ilex paraguariensis.

Table 1 - Collection sites of Ilex paraguariensis seeds.

\begin{tabular}{lccc}
\hline Lote & Altitude $(\mathbf{m})$ & $\begin{array}{c}\text { Pluviosidade média } \\
(\mathbf{m m} / \mathbf{a n o})\end{array}$ & Temperatura média $\left({ }^{\circ} \mathbf{C}\right)$ \\
\hline Porto União - SC & 780 & 1667 & 17,7 \\
Ajuricaba - RS & 331 & 1768 & 19,8 \\
Campo Erê - SC & 903 & 2045 & 16,7 \\
Urupema - SC & 1324 & 1634 & 14,1 \\
\hline
\end{tabular}

Fonte: Climate (2018)

Em cada lote, frutos maduros foram coletados de diversas matrizes, distanciadas em no mínimo 50 metros. As coletas foram realizadas por meio de anteparos de tecido colocados abaixo da copa das matrizes, não sendo utilizados frutos caídos ao chão. Foram considerados maduros, 
os frutos com coloração arroxeada-escura, classificados como cor 2,5/1 F: $5 Y$ da Tabela de Cores Munsell (MUNSELL, 2009).

As sementes foram extraídas dos frutos em peneira e água corrente, beneficiadas manualmente para retirada das impurezas e imediatamente utilizadas nas avaliações.

Para a realização do processo de estratificação, as sementes foram acondicionadas em caixas gerbox, em que uma camada de $0,25 \mathrm{~cm}$ de sementes ficou entre duas camadas de areia úmida com $1 \mathrm{~cm}$ cada. A areia foi autoclavada a $121^{\circ} \mathrm{C}$ por 20 minutos e umedecida até atingir $60 \%$ da capacidade de campo com água, conforme indicação das Regras para Análises de Sementes (BRASIL, 2009), sendo reumedecida periodicamente a cada três dias. A estratificação foi realizada segundo indicação de Cuquel, Carvalho e Chamma (1994). Cem sementes, por lote, permaneceram acondicionadas em alternância de temperatura de $15^{\circ} \mathrm{C}$ por 16 horas e $35^{\circ} \mathrm{C}$ por 8 horas durante três meses, e outras 100 sementes, por lote, durante o período de seis meses.

Depois do período de estratificação, as sementes foram submetidas ao teste de sanidade, no qual foram previamente desinfestadas com hipoclorito de sódio a $1 \%$ por 30 segundos e álcool $70 \%$ antes do plaqueamento, após foram lavadas três vezes em água destilada para remover o resíduo do hipoclorito de sódio.

Quatro repetições de $25(12+13)$ sementes foram dispostas em duas placas de Petri contendo meio de cultura BDA (Batata-Dextrose-Ágar) e acondicionadas a $25 \pm 2{ }^{\circ} \mathrm{C}$ em câmara climatizada com fotoperíodo de 12 horas, durante sete dias. Posteriormente, realizou-se a avaliação do teste, baseando-se na análise de estruturas fúngicas, com auxílio de microscópio estereoscópico e óptico. A identificação fúngica foi feita por meio de comparação com as características descritas em literatura específica (BARNETT; HUNTER, 1972). Para os gêneros Fusarium e Aureobasidium, a identificação foi realizada molecularmente, pelo Instituto Biológico. Foram identificados F. guttiforme, Fusarium oxysporum e Aureobasidium melanogenum, por meio da região TEF (Translation Elongation Fator) para Fusarium guttiforme e ITS (Internal Transcribed Spacer, não depositados no GenBank) para os demais. Novas colônias das espécies identificadas molecularmente foram avaliadas, posteriormente, pela comparação morfológica com as mesmas. Os dados de incidência dos fungos foram expressos em porcentagem.

$\mathrm{O}$ delineamento experimental utilizado foi o inteiramente casualizado. $\mathrm{O}$ arranjo dos dados do experimento de tratamento das sementes foi caracterizado como bifatorial (4 x 3), composto por quatro lotes e três períodos de estratificação (0,90 e 180 dias). Para a análise de variância, como os dados não apresentaram normalidade, foram transformados pela fórmula arcsen $[(x+0,5) /(100)]^{1 / 2}$. A comparação das médias para o fator lotes foi feita pelo teste de Tukey a $5 \%$ de probabilidade de erro e o programa utilizado para as análises estatísticas foi o SISVAR (FERREIRA, 2000). Já para o fator tempo de estratificação, os dados passaram por ajuste de regressão no Microsoft Excel $^{\oplus}$.

\section{Resultados e discussão}

Externamente, pode-se perceber que, ao final do período de estratificação, as sementes adquiriram coloração escurecida, em relação ao início do processo (Figura 1). Para Grigoletti Júnior et al. (1999), esta mudança na coloração das sementes se deve ao processo de decomposição do endocarpo, causado, na maioria das vezes, pela elevada umidade e ação de micro-organismos, como os fungos.

Pela análise de variância, foi verificada interação significativa entre o fatorial: lotes $\mathbf{x}$ períodos de estratificação (Tabela 2 e Figura 2). Foram observados valores de Coeficiente de Variação (CV) de 21,75\% a 60,87\% (Tabela 2). De acordo com Pimentel-Gomes e Garcia (2002), apesar de experimentos em condições bem controladas, como em laboratório, resultarem em baixos CV, isso não é regra geral, o que tem sido verificado em outros trabalhos com sanidade de sementes (AIMI et al., 2016; MEDEIROS et al., 2016). 
Figura 1 - Aspecto externo de sementes de erva-mate. (A) sementes não submetidas ao processo de estratificação; (B) sementes submetidas ao período de 180 dias de estratificação.

Figure 1 - External appearance of yerba mate seeds. (A) seeds not submitted to the stratification process; (B) seeds submitted to the 180-day stratification period.

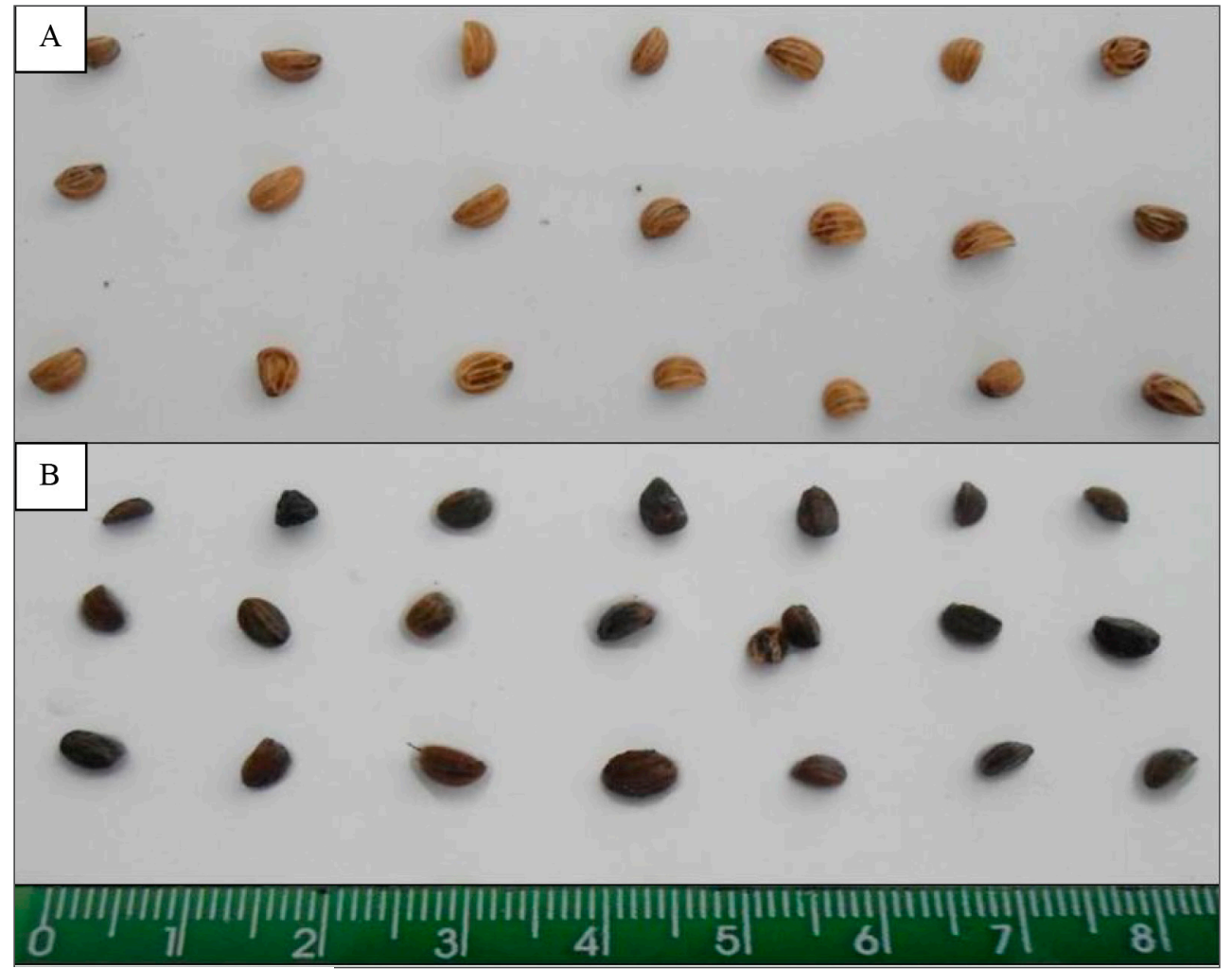

Fonte: Autores (2019)

Em sementes de erva-mate submetidas ao processo de estratificação, foram identificados os seguintes fungos: Fusarium graminearum (p value: 0,000 ), Fusarium oxysporum (p value: 0,001 ), Fusarium guttiforme (p value: 0,000 ), Paecilomyces sp. (p value: 0,005 ), Aspergillus sp. (p value: 0,0451) e Penicillium sp. (p value: 0,009). Foram observados ainda, em menor quantidade, Aureobasidium melanogenum, Trichoderma sp., Curvularia sp., Rhizopus sp., Alternaria sp., Cladosporium sp. e Bipolaris sp. (Tabela 2). Analisando sementes de erva-mate durante a estratificação, Grigoletti Júnior et al. (1999) identificaram os fungos Trichoderma sp., Fusarium sp., Rhizopus sp. e Alternaria sp.; além de Penicillium sp., Aspergillus sp., Curvularia sp., Cladosporium sp., Colletotrichum sp., Chaetomium sp. e Graphium sp. em menores quantidades.

De maneira geral, Fusarium spp. teve comportamento decrescente com relação à incidência em sementes de erva-mate ao longo do período de estratificação. Em alguns casos, foi observado um aumento aos 90 dias, porém, seguido de redução considerável aos 180 dias. Também foi verificada diminuição no desenvolvimento de suas colônias, tanto com relação ao tamanho como do desenvolvimento de coloração característica de cada espécie fúngica ao longo da estratificação (Tabela 2 e Figura 2). 
Efeito da estratificação em substrato esterilizado na qualidade sanitária de sementes... 858

Tabela 2 - Incidência fúngica (\%) em sementes de erva-mate de diferentes lotes e submetidas a diferentes períodos de estratificação.

Table 2 - Fungal incidence (\%) of yerba mate seeds from different lots, submitted to different periods of stratification.

\begin{tabular}{|c|c|c|c|c|c|}
\hline \multicolumn{6}{|c|}{ Período de estratificação (dias) } \\
\hline Fungo & Lote & 0 & 90 & 180 & Média \\
\hline \multirow{4}{*}{ 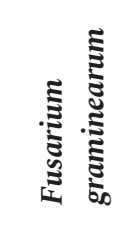 } & Porto União & $33,5 \mathrm{c}^{1}$ & $9,3 \mathrm{~b}$ & $1,0 \mathrm{a}$ & 14,6 \\
\hline & Campo Erê & $0,0 \mathrm{a}$ & $3,0 \mathrm{a}$ & $0,0 \mathrm{a}$ & 1 \\
\hline & Ajuricaba & $0,0 \mathrm{a}$ & $15,0 \mathrm{c}$ & $0,0 \mathrm{a}$ & 5 \\
\hline & Urupema & $9,0 \mathrm{~b}$ & $0,0 \mathrm{a}$ & $0,0 \mathrm{a}$ & 3 \\
\hline Média & & 10,6 & 6,8 & 0,3 & CV\%: 24,76 \\
\hline \multicolumn{6}{|c|}{ Período de estratificação (dias) } \\
\hline Fungo & Lote & 0 & 90 & 180 & Média \\
\hline \multirow{4}{*}{ 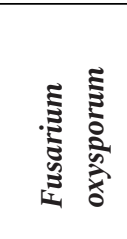 } & Porto União & $12,5 \mathrm{ab}$ & $12,7 \mathrm{ab}$ & $0,0 \mathrm{a}$ & 8,4 \\
\hline & Campo Erê & $22,5 \mathrm{~b}$ & $19,7 \mathrm{~b}$ & $63,5 \mathrm{~b}$ & 26,4 \\
\hline & Ajuricaba & $0,0 \mathrm{a}$ & $15,0 \mathrm{~b}$ & $0,0 \mathrm{a}$ & 5 \\
\hline & Urupema & $12,0 \mathrm{ab}$ & $0,0 \mathrm{a}$ & $0,0 \mathrm{a}$ & 4 \\
\hline Média & & 11,7 & 11,8 & 15,8 & CV\%: 21,75 \\
\hline \multicolumn{6}{|c|}{ Período de estratificação (dias) } \\
\hline Fungo & Lote & 0 & 90 & 180 & Média \\
\hline \multirow{4}{*}{ 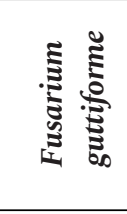 } & Porto União & $22,0 \mathrm{~b}$ & $21,7 \mathrm{c}$ & $0,5 \mathrm{a}$ & 14,7 \\
\hline & Campo Erê & $30,5 \mathrm{~b}$ & $13,0 \mathrm{~b}$ & $1,0 \mathrm{a}$ & 14,8 \\
\hline & Ajuricaba & $4,0 \mathrm{a}$ & $29,5 \mathrm{c}$ & $0,0 \mathrm{a}$ & 11,1 \\
\hline & Urupema & $27,0 \mathrm{~b}$ & $8,0 \mathrm{a}$ & $0,0 \mathrm{a}$ & 11,6 \\
\hline Média & & 20,9 & 18,1 & 0,3 & CV\%: 27,49 \\
\hline \multicolumn{6}{|c|}{ Período de estratificação (dias) } \\
\hline Fungo & Lote & 0 & 90 & 180 & Média \\
\hline \multirow{4}{*}{ 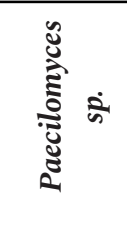 } & Porto União & $0,0 \mathrm{a}$ & $0,0 \mathrm{a}$ & $81,0 \mathrm{~b}$ & 27 \\
\hline & Campo Erê & $0,0 \mathrm{a}$ & $0,0 \mathrm{a}$ & $7,5 \mathrm{~b}$ & 2,5 \\
\hline & Ajuricaba & $0,0 \mathrm{a}$ & $0,0 \mathrm{a}$ & $0,0 \mathrm{a}$ & 0 \\
\hline & Urupema & $0,0 \mathrm{a}$ & $0,0 \mathrm{a}$ & $0,0 \mathrm{a}$ & 0 \\
\hline Média & & 0 & 0 & 22,1 & CV\%: 24,20 \\
\hline \multicolumn{6}{|c|}{ Período de estratificação (dias) } \\
\hline Fungo & Lote & 0 & 90 & 180 & Média \\
\hline \multirow{4}{*}{ 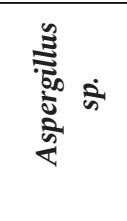 } & Porto União & $1,5 \mathrm{a}$ & $0,0 \mathrm{a}$ & $0,5 \mathrm{a}$ & 0,7 \\
\hline & Campo Erê & $2,0 \mathrm{a}$ & $0,0 \mathrm{a}$ & $0,0 \mathrm{a}$ & 0,7 \\
\hline & Ajuricaba & $0,0 \mathrm{a}$ & $6,5 \mathrm{~b}$ & $0,0 \mathrm{a}$ & 1,6 \\
\hline & Urupema & $0,0 \mathrm{a}$ & $5,5 \mathrm{ab}$ & $0,0 \mathrm{a}$ & 1,8 \\
\hline Média & & 0,9 & 3 & 0,1 & CV\%: 60,87 \\
\hline
\end{tabular}


Tabela 2 - Conclusão...

Table 2 - Conclusion...

\begin{tabular}{|c|c|c|c|c|c|}
\hline \multicolumn{6}{|c|}{ Período de estratificação (dias) } \\
\hline Fungo & Lote & 0 & 90 & 180 & Média \\
\hline \multirow{4}{*}{ 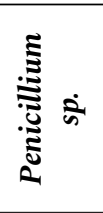 } & Porto União & $3,0 \mathrm{a}$ & $0,0 \mathrm{a}$ & $0,0 \mathrm{a}$ & 1 \\
\hline & Campo Erê & $9,0 \mathrm{~b}$ & $0,0 \mathrm{a}$ & $0,0 \mathrm{a}$ & 3 \\
\hline & Ajuricaba & $3,0 \mathrm{a}$ & $0,0 \mathrm{a}$ & $0,5 \mathrm{a}$ & 1,2 \\
\hline & Urupema & $2,5 \mathrm{a}$ & $1,0 \mathrm{a}$ & $7,5 \mathrm{~b}$ & 3,7 \\
\hline Média & & 4,4 & 0,3 & 2 & CV\%: 50,09 \\
\hline
\end{tabular}

Fonte: Autores (2019)

${ }^{1}$ Letras maiúsculas iguais para linhas e minúsculas para colunas não diferem estatisticamente entre si pelo teste de Tukey a $5 \%$ de probabilidade.

Figura 2 - Incidência fúngica em sementes de erva-mate de diferentes lotes e submetidas a diferentes períodos de estratificação.

Figure 2 - Fungal incidence of yerba mate seeds from different lots, submitted to different periods of stratification.

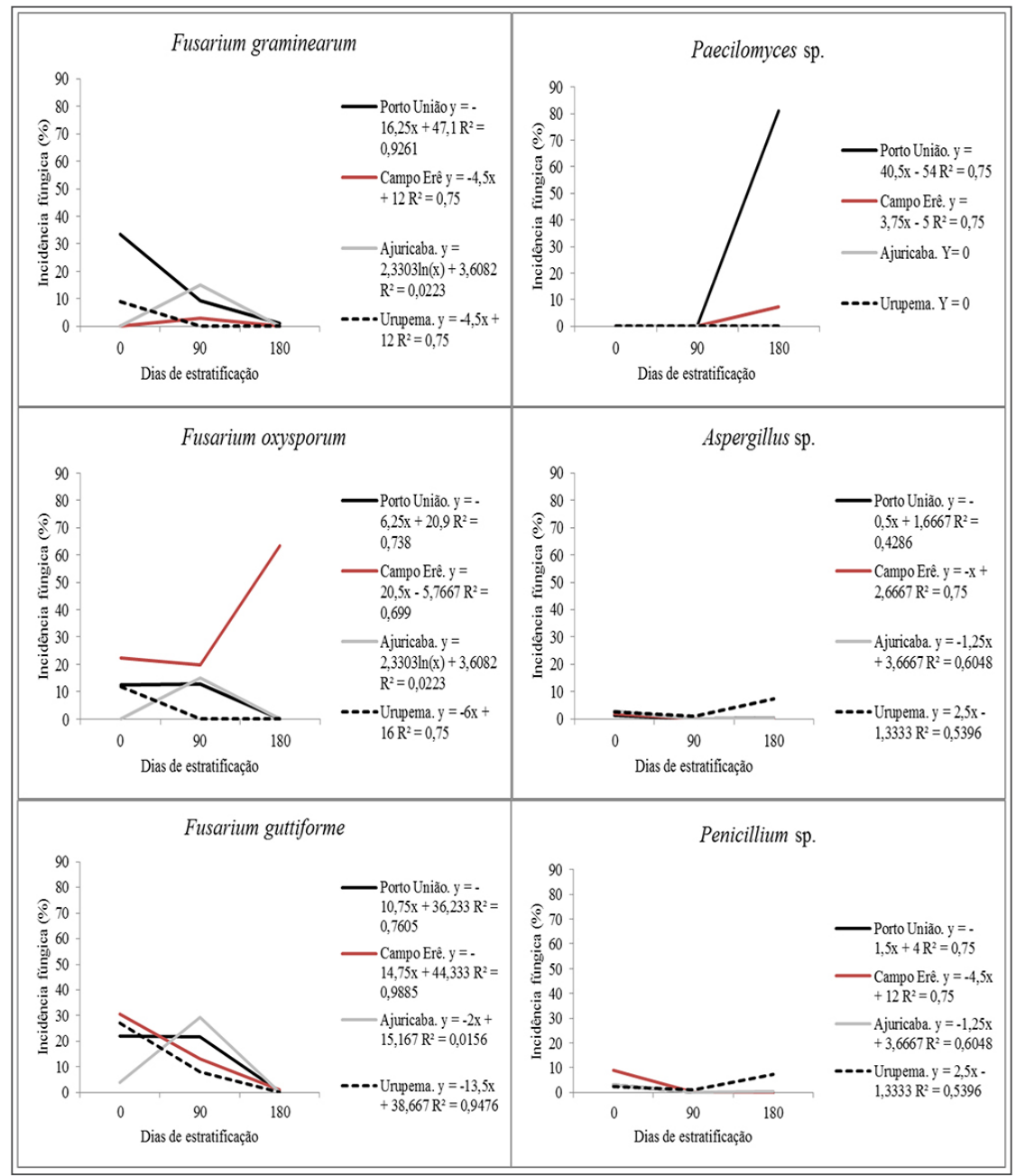

Fonte: Autores (2019) 
O lote de Porto União teve a maior incidência inicial para Fusarium graminearum (33,5\%), sendo que se observa uma redução considerável deste fungo, para o lote em análise, na medida em que se aumenta o período de estratificação. Essa redução também é verificada para o lote de sementes oriundo de Urupema. Já o lote de Ajuricaba não apresentou Fusarium graminearum inicialmente (sem estratificação), percebendo-se um aumento deste no terceiro mês (90 dias) de estratificação e não houve incidência no sexto mês do processo (120 dias) (Tabela 2 e Figura 2).

Com relação ao fungo Fusarium oxysporum, a maior ocorrência se deu no lote de Campo Erê (22,5\%), sendo que houve um crescimento significativo na sua observação aos seis meses de estratificação (63,5\%). Para o lote de Ajuricaba, F. oxysporum teve ocorrência apenas no terceiro mês (90 dias) de avaliação. Para os demais lotes, percebe-se uma tendência de diminuição do fungo ao longo do período de estratificação (Tabela 2).

De maneira geral, Fusarium guttiforme teve as maiores ocorrências iniciais para os lotes analisados, com exceção de Ajuricaba que apresentou apenas $4 \%$ de incidência. Foi observado um padrão de diminuição de Fusarium guttiforme ao longo do período de estratificação, com ocorrências insignificantes aos 180 dias do processo de superação de dormência (Tabela 2 e Figura 2).

Grigoletti Júnior et al. (1999) verificaram o aumento de ocorrência fúngica em sementes estratificadas até 150 dias do processo, seguido por uma redução de ocorrência aos 180 dias. Os mesmos autores citam que esse fato se deve à redução de nutrientes existentes no substrato utilizado para a estratificação, sendo que os autores não utilizaram areia esterilizada. No presente trabalho, a redução fúngica ao longo da estratificação pode estar relacionada à diminuição de nutrientes das sementes, já que o substrato esterilizado não possui nutrientes disponíveis.

Poletto et al. (2015) avaliaram sementes no final do processo de estratificação realizado por diversos viveiristas, e verificaram uma contaminação fúngica por Fusarium sp. e Rhizoctonia sp. próxima a $100 \%$ das sementes avaliadas. Os mesmos autores relataram que geralmente os viveiristas não realizam a desinfestação do substrato utilizado e muitas vezes acabam reutilizando este material; podendo-se inferir, então, que grande parte da contaminação gerada pode ser oriunda de substratos e materiais contaminados que são utilizados em viveiros. Como verificado no presente trabalho, a utilização de um substrato esterilizado pode ser bastante eficiente no controle fúngico durante a estratificação de sementes de erva-mate.

Nenhum dos lotes avaliados apresentou Paecilomyces sp. inicialmente. Sua ocorrência se deu aos 180 dias de estratificação para os lotes de Porto União (81\%) e Campo Erê (7,5\%) (Tabela 2). Poletto et al. (2015) verificaram a presença de Paecilomyces sp. em flores, frutos e sementes despolpadas por viveiristas, assim como em sementes estratificadas de erva-mate. Este gênero é considerado saprofítico e apresenta capacidade de produção de enzimas celulolíticas (BASSO; GALLO; BASSO, 2010; LOPES et al., 2016), fazendo com que este fungo possa atuar no processo de degradação do endocarpo e do tegumento das sementes de erva-mate, auxiliando no processo de superação de dormência. Esse fungo também foi relatado em sementes de dendê (CYSNE; SOUZA; LIMA, 2015), Myracrodruon urundeuva (BARBOSA et al., 2014) e Amburana cearenses (PINHEIRO et al., 2014).

As incidências iniciais de Aspergillus sp. foram de no máximo $2 \%$ para o lote de Campo Erê, se mantendo baixas ao longo do processo de estratificação; no entanto, os lotes Ajuricaba e Urupema tiveram aumentos significativos aos 90 dias de estratificação. O mesmo se verifica para Penicillium sp. que somente apresentou aumento significativo para o lote de Urupema aos 180 dias de estratificação (Tabela 2 e Figura 2). Os gêneros Aspergillus e Penicillium são considerados fungos de armazenamento, podem causar o apodrecimento de sementes de diversas espécies, ocasionando perdas no poder germinativo, descoloração parcial ou total de sementes, aquecimento, transformações bioquímicas, produção de toxinas e modificações celulares (WEITZEL, 1987). Geralmente em condições com elevada umidade e grande concentração de sementes, como é o caso da estratificação, estes gêneros apresentam grande incidência. 
Para todos os fungos avaliados, os diferentes comportamentos de incidência ao longo da estratificação podem estar relacionados ao fator qualidade inicial de sementes, sendo que sementes com menor qualidade apresentam maior predisposição ao ataque de fungos (WEITZEL, 1987); além disso, a utilização de substrato esterilizado pode contribuir no controle fúngico de sementes de erva-mate.

\section{Conclusões}

A incidência de Fusarium graminearum, Fusarium oxysporum, Fusarium guttiforme, Aspergillus sp. e Penicillium sp. em sementes de erva-mate diminuiu após a estratificação em areia autoclavada por um período de 180 dias.

O gênero Paecilomyces apresentou incidência somente aos 180 dias de estratificação, podendo estar relacionado com a degradação do endocarpo de sementes de erva-mate.

\section{Agradecimentos}

A Fundação de Amparo à Pesquisa e Inovação do Estado de Santa Catarina (FAPESC).

\section{Referências}

AIMI, S. C. et al. Teste de sanidade e germinação em sementes de Cabralea canjerana (Vell.) Mart. Ciência Florestal, Santa Maria, v. 26, n. 4, p. 1361-1370, 2016.

BARBOSA, L. G. et al. Micoflora de sementes de aroeira (Myracrodruon urundeuva Fr. All) submetidas a diferentes temperaturas. In: WORKSHOP DE SEMENTES E MUDAS DA CAATINGA, 4., 2014. Anais [...]. Petrolina: EMBRAPA Semiárido, 2014. p. 23-27.

BARNETT, H. L.; HUNTER, B. B. Illustred genera of imperfect fungi. $3^{\text {th }}$ ed. Minnesota: Burgess, 1972.

BASSO, T. P.; GALLO, C. R.; BASSO, L. C. Atividade celulolítica de fungos isolados de bagaço de cana-de-açúcar e madeira em decomposição. Pesquisa Agropecuária Brasileira, Brasília, v. 45, n. 11, p. 1282-1289, 2010.

BEWLEY, J. D. et al. Seeds: physiology of development, germination and dormancy. $3^{\text {th }}$ ed. New York: Springer, 2013.

BRASIL. Regras para análise de sementes. Brasília: Mapa; ACS, 2009. 399 p.

CLIMATE. Dados Climáticos para cidades mundiais. Oedheim, [2018]. Disponível em: http:// pt.climate-data.org/. Acesso em: 09 set. 2016.

CROCE, D. M. da; FLOSS, P. A. Cultura da erva-mate no Estado de Santa Catarina. Florianópolis: EPAGRI, 1999. $81 \mathrm{p}$.

CUQuel, F. L.; CARVAlho, M. L. M.; ChammA, H. M. C. P. Avaliação de métodos de estratificação para a quebra de dormência de sementes de erva-mate. Scientia Agrícola, Piracicaba, v. 51, n. 3, p.415-421, 1994.

CYSNE, A. Q.; SOUZA, M. G.; LIMA, W. A. A. Fungos associados a sementes híbridas interespecíficas de dendê em função da assepsia e do beneficiamento. Amazonian Journal of Agricultural and Environmental Sciences, Belém, v. 58, n. 4, p. 372-378, 2015.

DANIEL, O. Erva-mate: sistema de produção e processamento industrial. Dourados: Editora UFGD, 2009. 288 p. 
FERREIRA, D. F. Análises estatísticas por meio do Sisvar para Windows versão 4.0. In: REUNIÃO ANUAL DA REGIÃO BRASILEIRA DA SOCIEDADE INTERNACIONAL DE BIOMETRIA, 45., 2000. Anais [...]. São Carlos: SIB, 2000. p. 255-258.

FOWLER, J. A. P.; BIANCHETTI, A. Dormência em sementes florestais. [S. l.]: EMBRAPA Florestas, 2000. 27 p. (Documentos, 40).

GRigoletti JUnior, A.; AUER, C. G. Doenças da erva-maten: identificação e controle. Colombo: EMBRAPA; CNPF, 1996.

GRIGOLETTI JUNIOR, A. et al. Efeito de fungicidas aplicados nas sementes, na emergência de plântulas de erva-mate (Ilex paraguariensis St. Hil.). Boletim de Pesquisa Florestal, Colombo, n. 39, p. 31-39, jul./dez. 1999.

LOPES, A. M. et al. Primeiro relato da ocorrência de Paecilomyces formosus e Paecilomyces parvisporus no Brasil. Revista Brasileira de Biociências, Porto Alegre, v. 14, n. 4, p. 215-224, 2016.

MARCOS FILHO, J. Fisiologia de sementes de plantas cultivadas. Piracicaba: FEALQ, 2005.

MEDEIROS, J. G. F. et al. Fungos associados às sementes de Enterolobium contortisiliquum: Análise da incidência, controle e efeitos na qualidade fisiológica com o uso de extratos vegetais. Ciência Florestal, Santa Maria, v. 26, n. 1, p. 47-58, 2016.

MUNSELL soil color charts: with genuine Munsell color chips. Grand Rapids: Munsell Color, 2009. v. 1.

PIMENTEL-GOMES, F.; GARCIA, C. H. Estatística aplicada a experimentos agronômicos e florestais exposição com exemplos e orientações para uso de aplicativos. Piracicaba: FEALQ, 2002. 309 p.

PINHEIRO, G. S. et al. Micoflora Fúngica de sementes de umburana-de-cheiro (Amburana cearenses (Arr. Cam.) A.c Smith). In: WORKSHOP DE SEMENTES E MUDAS DA CAATINGA, 4., 2014. Anais [...]. Petrolina: EMBRAPA Semiárido, 2014. p. 137-140.

POLETTO, I. et al. Aspectos epidemiológicos da podridão-de-raízes da erva-mate (Ilex paraguariensis). Ciência Florestal, Santa Maria, v. 25, n. 2, p. 281-291, abr./jun. 2015.

WEITZEL, M. M. V. S. Fungos de armazenamento. In: SOAVE, J.; WETZEL, M. M. S. Patologia de Sementes. Campinas: Fundação Cargill, 1987. p. 260-275. 